\title{
Theory of coherent molecule to surface electron injection: An analytical model ${ }^{\dagger}$
}

\author{
S RAMAKRISHNA ${ }^{1}$, T SEIDEMAN ${ }^{1, *}$, F WILLIG $^{2}$ and V MAY $^{3}$ \\ ${ }^{1}$ Department of Chemistry, Northwestern University, Evanston, IL 60208, USA \\ ${ }^{2}$ Fritz-Haber-Institut der MPG, Faradayweg 4-6, 14195 Berlin, Germany \\ ${ }^{3}$ Institut für Physik, Humboldt-Universität, AG Photobiophysik, Newtonstrasse 1512489 Berlin, \\ Germany \\ e-mail: t-seideman $a$ northwestern.edu
}

\begin{abstract}
Electron transfer from a molecular level to empty continuum levels of a substrate is described theoretically. Using a quasicontinuum approach to model the substrate, analytical expressions pertaining to the time-dependent probability among the various levels of the substrate is presented along with its extension to coherently excited molecular vibrational modes. Hidden time scales and dynamics are revealed in the analysis and possible experiments to observe the new results are suggested. We note the applicability of the model to the description of a variety of other phenomena that are formally similar to the electron injection problem, although pertaining to different physics.
\end{abstract}

Keywords. Heterogeneous electron transfer; photoinduced ultrafast dynamics; vibrational wavepackets.

\section{Introduction}

Electron injection from a molecular level into unoccupied levels of a metallic or semiconductor substrate is a basic process that underlies several sub-fields of interfacial science, including electrochemistry, ${ }^{1}$ surface photochemistry ${ }^{2}$ and molecular electronics. ${ }^{3}$ Photoinduced electron transfer (ET) from excited electronic states of molecules attached to semiconductor surfaces, such as $\mathrm{TiO}_{2}$, is an ideal model to study the basic physics underlying electron injection processes, ${ }^{5}$ and is also the first step in the operation of dye-sensitized solar cells. Usually a pump laser pulse initiates the injection process and a probe pulse that follows it at a variable time delay, can time-resolve either the decay of the molecular donor or the formation of the molecular cation. The decay of the initial molecular donor state is known to depend on factors such as: (i) the electronic overlap between the donor and the substrate electronic levels, (ii) the substrate density of states, (iii) the molecular reorganization energy that occurs when the nuclear modes shift their equilibrium position due to ionization, and (iv) the energetic position of the molecular donor level relative to the conduction band edge of the semiconductor. ${ }^{4}$ Femtosecond pump-probe

\footnotetext{
${ }^{\dagger}$ Dedicated to the memory of the late Professor S K Rangarajan
} *For correspondence experiments have revealed that photoinduced heterogeneous electron transfer from a molecule to a semiconductor occurs before full vibrational relaxation of the excited molecule is achieved. ${ }^{5,6}$ Accordingly, theoretical description of the decay of the initial state has been formulated to include vibrational coherence in the transfer process. ${ }^{4}$

\section{Analytical model}

Although the theoretical study of the decay of an initially populated molecular donor state into a final continuum of electronic levels has had a long history, ${ }^{4,7-9}$ the rise of the injected population among the various continuum electronic levels has been studied only recently, revealing Franck-Condon dictated structures in the ultrafast time regime that can be influenced by laser pulse-induced vibrational coherences. ${ }^{10-12}$ In this article, we present analytical expressions for the rise of probability among a uniform quasicontinuum (QC) of electronic states, which represents the substrate, from an initially populated discrete electronic state. In the process what is achieved is a generalization of the wellknown results of the Wigner-Weisskopf ${ }^{13}$ and Fano-Anderson ${ }^{14}$ models (which deal with the static broadening of a discrete electronic level interacting with a continuum of levels), to the entire time 
domain, inclusive of coherent and incoherent molecular vibrational degrees of freedom.

In general, the injected distribution will relax mainly due to interactions with phonons or electronelectron interactions. ${ }^{15}$ These coherence limiting processes are not discussed here, since our purpose is to understand the basic physics of the injection process, but we note that the theory applies at low injection densities and short time scales.

The Hamiltonian describing the coupled vibrational-electronic dynamics is given as

$$
H=\sum_{a} H_{a}\left|\varphi_{a}\right\rangle\left\langle\varphi_{a}\right|+\sum_{k}\left(V_{k e}|| \varphi_{-k}\right\rangle\left\langle\left\langle\varphi_{-e}\right|+\text { h.c. }\right),
$$

where $\left|\varphi_{a}\right\rangle$ are the electronic states and $Q_{\xi}$ denotes the set of vibrational coordinates participating in the ET process. The $Q_{\xi}$ are governed by the vibrational Hamiltonian $H_{a}$, where the electronic quantum number a includes the molecular excited state, $a=e$ and the QC of band states, $a=k$. The transfer integral $V_{e k}$ couples the electron in the excited donor state to the semiconductor QC. One can account for the photoexcitation by introducing an additional molecular electronic state (the ground state), which is coupled to the excited state $\left|\varphi_{e}\right\rangle$ via an electric field. ${ }^{10}$ In the impulsive excitation limit, where the pulse duration of the excitation laser is much shorter than all system time scales, such as the ET and vibrational times, the photoexcitation process can be ignored and the initial state taken to be the excited state. In what follows we neglect also excitation pathways that proceed directly from the ground molecular state to the QC and can give rise to Fano interferences. $^{16}$

The system dynamics is obtained by solving the time dependent Schrödinger equation for the electronic-vibrational wavefunction $|\psi(t)\rangle=$ $\sum_{a v} A_{a v}(t)\left|\chi_{a v}\right\rangle\left|\varphi_{a}\right\rangle$, where $v$ is a vibrational index and $\left|\chi_{a v}\right\rangle$ is the corresponding eigenstate of the vibrational (Born-Oppenheimer) Hamiltonian governing the dynamics in state $a$. Here $A_{a v}(t), a=e, k$ are time-dependent expansion coefficients whose square moduli give the probabilities of interest.

\section{Dynamics of the injected electron}

It is instructive to start the discussion by neglecting the vibrational degrees of freedom and focusing on the electronic dynamics. It will be shown that such a description contains the essential physics of ET from a discrete to a continuum set of electronic states, but the vibrational dynamics introduces interesting new phenomena. With the vibrational quantum numbers omitted, the equation of motion for the expansion coefficient of each level $k$ of the QC is given as

$$
A_{k}(t)=-i / \hbar \int_{0}^{t} V e^{i\left(\varepsilon_{k}-\varepsilon_{e}\right) t^{\prime} / \hbar} A_{e}\left(t^{\prime}\right) \mathrm{d} t^{\prime},
$$

where the electronic coupling $V_{k e}$ has been set to $V$, a constant, and $\varepsilon_{a}$ denote the eigen-energies of the electronic states. In the wide band limit, where the excited state is coupled with a large, uniformly spaced QC of electronic levels, $A_{e}(t)$ reduces to,

$$
A_{e}(t)=A_{e}(t=0) e^{-\pi|V|^{2} \rho t \hbar},
$$

signifying that the molecular occupation probability $\left|A_{e}(t)\right|^{2}$ decays exponentially due to interaction with the quasi continuum of electronic levels. In (3), $\rho^{-1}$ is the energy spacing between the QC levels. It should be noted that only the continuum aspect of the decay is present in (3); the recurrences that set in at times much longer than the ET timescale, due to the discrete nature of the QC, are not accounted for. ${ }^{4}$ Since the transfer time-scale depends on the product $|V|^{2} \rho$, it is convenient to renormalize $V$ such that the transfer process is studied independent of the level spacing $\rho^{-1}{ }^{4}$ Using (3), the time dependent occupation probability of the continuum states is given as

$$
\begin{aligned}
& \left|A_{k}(t)\right|^{2}=\frac{|V|^{2}\left|A_{e}(t=0)\right|^{2}}{\left(\varepsilon_{k}-\varepsilon_{e}\right)^{2}+\left(\pi|V|^{2} \rho\right)^{2}} \\
& \left(1-2 \times \cos \left[\left(\varepsilon_{k}-\varepsilon_{e}\right) t / \hbar\right] e^{-\pi|V|^{2} \rho t / \hbar}+e^{-2 \pi|V|^{2} \rho t \hbar}\right) .
\end{aligned}
$$

The above result has also been obtained using a Green's function approach. ${ }^{11}$ It is immediately apparent from (4) that the time-dependent rise of probability in a level $k$ of the QC exhibits two time constants, unlike the decay of the discrete state, which exhibits a pure exponential decay subject to a single time-scale $\tau_{e}=\hbar / 2 \pi|V|^{2} \rho$. The slower time constant in (4) is modulated by an oscillatory cosine term, whose frequency depends on the energy difference between the initial discrete and the final QC state. Physically this term is related to the phenomenon of quantum beats that is observed when two or more states are excited coherently. In the present case the oscillations are damped owing to the interactions with a dense final manifold. At times long with respect 
to the ET times $\tau_{e}$, the time dependence is minimal, and asymptotically the probability converges to

$$
\lim _{t \rightarrow \infty}\left|A_{k}(t)\right|^{2}=\frac{|V|^{2}\left|A_{e}(t=0)\right|^{2}}{\left(\varepsilon_{k}-\varepsilon_{e}\right)^{2}+\left(\pi|V|^{2} \rho\right)^{2}} .
$$

Equation (5) describes a Lorentzian function with a FWHM of $2 \pi||^{2} \rho$ and is readily recognized as the result of the Wigner-Weisskopf and Fano-Anderson models.

In figure 1, where the results of (4) are plotted vs the quasi continuum energy, it can be seen that at early times the injected distribution is quite broad, with ripples that signify quantum beats. At $t>\tau_{e}$, however, it converges to the density of states of the discrete level, which has now broadened into a Lorentzian due to its interaction with the continuum of electronic levels. The analytical expression given by (4), is in perfect agreement with results of independent numerical calculations. One is able to grasp the essential physics of the injection process from (4), while struck by the presence of the slower time constants and oscillatory terms, which are absent in the decay of the initial state. These features arise from our energy resolution of the continuum. The total probability in the continuum, $P_{Q C}=\sum_{k}\left|A_{k}(t)\right|^{2}$, is estimated in the limit of a dense manifold $\left(\rho^{-1} \rightarrow 0\right)$ by replacing the discrete summation by continuum integration over $\varepsilon_{k}$. Using (4) we find, $P_{\mathrm{QC}}=$ $\left|A_{e}(t=0)\right|^{2}\left(1-e^{-2 \pi \mid \eta^{2} \rho t / \hbar}\right)$, as expected from the requirement of population conservation. Hence the quantum beats and the slow rise time scales manifest only for individual levels or for a partial summation of $k$ levels in the QC. This is illustrated in figure 2, where partial summation over $k$ is performed, cen-

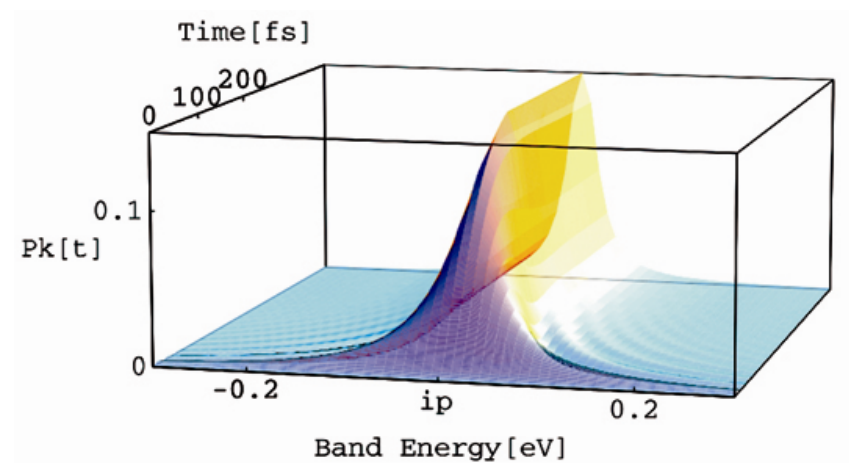

Figure 1. Rise of probability among the electronic levels of a QC of total width of $1.4 \mathrm{eV}$ due to injection from a discrete electronic level $\varepsilon_{e}$ positioned at energy $i_{p}$ in relation to the band energies. The plot has been obtained using (4). tered about different energies. It is seen that partial summation of the $\left|A_{k}(t)\right|^{2}$ over a range centered about the excited state energy $\varepsilon_{e}$ - the injection energy gives rise, as expected, to a population build up that is slower when the summation range is narrower, but in any case smooth, exhibiting no beats. By contrast, summation about a center other than $\varepsilon_{e}$ breaks the symmetry and gives rise to a beat pattern.

Experimental observation of the phenomena predicted here is, in principle, possible with current surface sensitive techniques, such as two photon photoemission (2PPE) ${ }^{17,18}$ With a pump probe to populate the excited state and a probe beam to photoionize the substrate, it is currently possible to energy- and time-resolve both the slower rise and the quantum beats, since the time- and energyresolutions required to that end are within the reach of present technology, at least in as far as the present model suggests.

The decay of the occupation probability of the molecular excited state including vibrational coherences has been derived and extensively discussed, previously. ${ }^{4}$ We present here approximate analytical expression for the probability of populating individual $k$-levels of the QC when vibrational modes are introduced. The decay pattern of the time dependent coefficient associated with each vibrational level of the initial molecular donor electronic state can be approximated as

$$
A_{e v}(t) \approx A_{e v}(t=0) \exp \left(-K_{v} t / 2\right),
$$

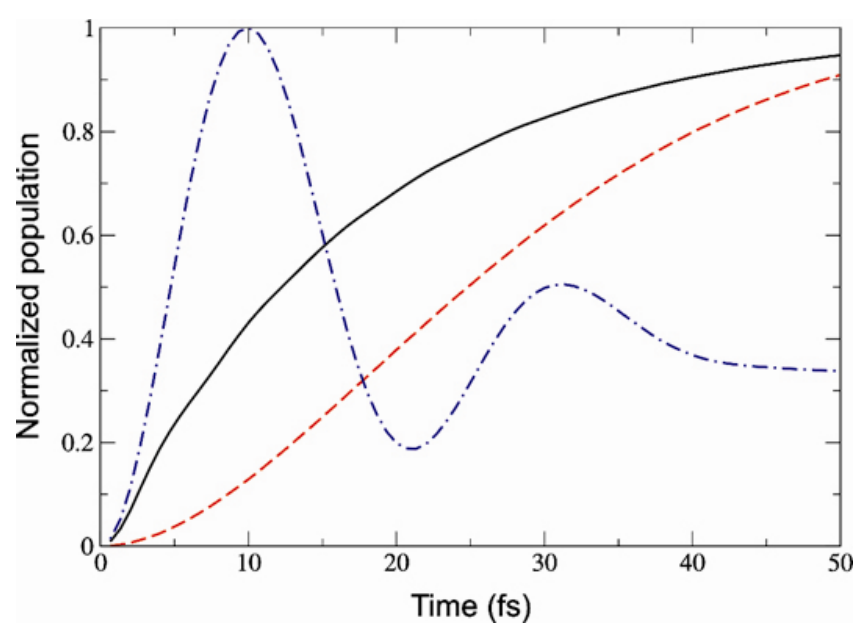

Figure 2. Rise of normalized total population versus time for the case considered in figure 1. Solid (black) curve indicates population summed over the entire QC $(1.4 \mathrm{eV})$ having a transfer time of $17 \mathrm{fs}$. Dashed (red) curve denotes population summed over an energy range of $100 \mathrm{meV}$ centered about the energy of $\varepsilon_{e}$, and dashdotted (blue) curve corresponds to population summed over a $100 \mathrm{meV}$ range centered $20 \mathrm{meV}$ above $\varepsilon_{e}$. 
whereby the QC coefficients are given as

$$
\begin{aligned}
& \left.A_{-k}(t)\right|^{2} \approx \sum_{\mu, v}|V|^{2}|\langle v \mid \mu\rangle|^{2}\left|A_{e v}(t=0)\right|^{2} \\
& \times \frac{1-2 \cos \left[\left(\varepsilon_{k \mu}-\varepsilon_{e v}\right) t / \hbar\right] e^{-K_{v} t / 2}+e^{-K_{v} t}}{\left(\varepsilon_{k \mu}-\varepsilon_{e v}\right)^{2}+\left(K_{v} \hbar / 2\right)^{2}} \\
& +\operatorname{Re}\left[\sum_{\mu} \sum_{v \neq v^{\prime}}\langle v \mid \mu\rangle\left\langle\mu \mid v^{\prime}\right\rangle A_{e v}(t=0) A_{e v^{\prime}}(t=0)\right. \\
& \times\left(\frac{1-e^{\left[-i\left(\varepsilon_{k \mu}-\varepsilon_{e v^{\prime}}\right)-K_{\nu^{\prime}} \hbar / 2\right] / \hbar}}{i\left(\varepsilon_{k \mu}-\varepsilon_{e v^{\prime}}\right)-K_{v^{\prime}} \hbar / 2}\right) \\
& \left.\times\left(\frac{1-e^{\left[-i\left(\varepsilon_{k \mu}-\varepsilon_{e v}\right)-K_{\nu} \hbar / 2\right] t \hbar \hbar}}{i\left(\varepsilon_{k \mu}-\varepsilon_{e v}\right)-K_{v} \hbar / 2}\right)\right] \text {. }
\end{aligned}
$$

In (7), $v$ and $\mu$ denote the excited state and QC vibrational indices, respectively, $\varepsilon_{e v}$ and $\varepsilon_{k \mu}$ are the excited state and QC vibronic energies, respectively and

$$
K_{v}=\frac{2 \pi}{\hbar} \sum_{\mu}|V|^{2}|\langle v \mid \mu\rangle|^{2} \rho,
$$

is the rate of transfer from vibrational level $v$ of the initial electronic state. The first term of (7) consists of the diagonal elements and has the same mathematical structure and physical content as the purely electronic probability of (4). The second term, consisting of the off-diagonal elements, reflects the coherences in the initially prepared excited manifold.

In the absence of coherences in the excitation, the second term disappear and our solution reduces to the result of ref. 11. Assuming that a single vibrational level $v_{\mathrm{o}}$ is initially occupied, one obtains a series of equally-spaced peaks for the occupation probabilities in the QC, spaced by the energy of the vibrational mode. The peaks are centered at values $\varepsilon_{k \mu}=\varepsilon_{e v_{0}}$ and their heights are determined by the Franck-Condon terms $\left|\left\langle v_{0} \mid \mu\right\rangle\right|^{2}$. Each peak is akin to what has already been analysed in the context of the pure electronic case, both with regard to the electronic quantum beats observed off the center of the peak and with regard to the decrease of the energetic spread of probability distribution as time increases. In case the initially excited state is a coherent vibrational wave packet, the second term of (7) is nonzero, leading to interferences that are manifested as additional oscillations, as seen along the midpositions of the peaks in figure 3. A slower time scale of 85 fs has been chosen for figure 3 , so that one can clearly resolve the individual Franck-Condon peaks already at early times. For the much shorter rise time considered in figure 1, one would obtain a broader and unstructured distribution, which persists until later times. These results explain analytically and generalize the numerical observations of (ref. 10). The phase of these oscillations depends on the overall sign of the product of the two Franck-Condon terms appearing in the off-diagonal term. As one moves down the QC band, the phase of the oscillations changes sign and this can also be understood in terms of wavepacket dynamics in the excited state. For $k$ states whose potential energy surfaces (PESs) cross that of the excited state at opposite ends of the classical turning points of the excited state vibrational wavepacket, the rise of probability will exhibit oscillations that differ in phase by a factor $\pi .^{10}$ Thus, the injected electronic distribution arising from a molecular level maps the initially excited coherent vibrational wavepacket: it bears the signature of molecular vibrational frequencies in the spacing of the peaks and exhibits the imprint of vibrational coherence as oscillations along the rise. In addition, the phase of these oscillations for the various $k$ levels of the QC contains information about the location of the excited state vibrational wavepacket with regard to the crossings between the initial and final molecular PESs. If the width of the uniform QC is sufficient to encompass the entire injected distribu-

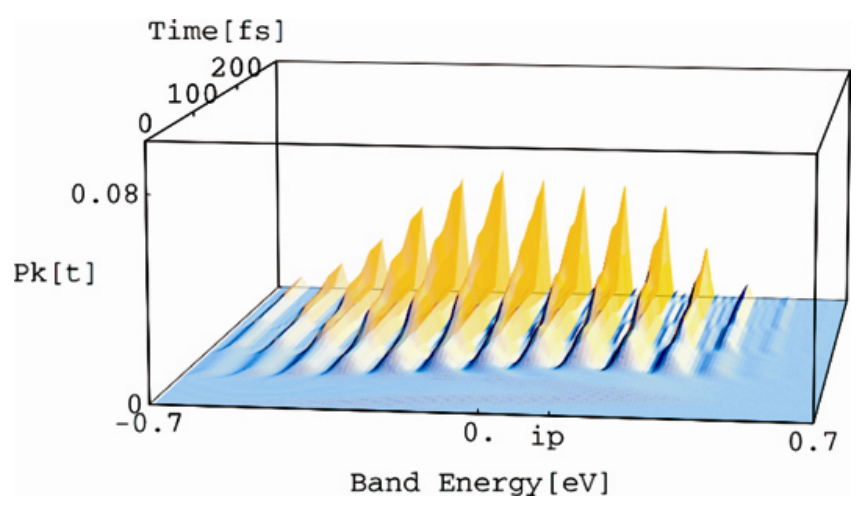

Figure 3. Time-dependent probability distribution versus the energy of a QC whose width and spacing are similar to figure 1 . The position of the injecting molecular level, $\varepsilon_{e}$, in the energy band of the substrate is indicated as $i_{p}$. A single harmonic vibrational mode of energy $0.1 \mathrm{eV}$ and an overall transfer time of around $85 \mathrm{fs}$ are inputs to (7) which has been used in the calculation. 
tion (wide-band limit), as in figure 3 , the total injected distribution will display a smooth rise due to fully destructive interference; otherwise it shows a step-like rise. In the wide-band limit all relevant Franck-Condon transitions from every initially populated vibrational state $(v)$ to all possible final vibrational states $(\mu)$ are fulfilled and hence $K_{v}$ reduces to the pure electronic limit $K_{v} \equiv K=$ $(2 \pi / \hbar)|V|^{2} \rho{ }^{4}$

The time-dependent probabilities of individual vibrational states of the ionized molecule can be obtained via (7) by recasting the total probabilities as $\left|A_{k}(t)\right|^{2}=\sum_{\mu l}\left|A_{k \mu}(t)\right|^{2}$ and summing the $\left|A_{k \mu t}(t)\right|^{2}$ over $k$. The rise of probability of level $\mu$ level of the ionized molecule thus takes the form,

$$
\begin{aligned}
& \left|A_{\mu}(t)\right|^{2}=\sum_{k}\left|A_{k \mu}(t)\right|^{2} \\
& \approx \sum_{k v}|V|^{2}|\langle v \mid \mu\rangle|^{2}\left|A_{e v}(t=0)\right|^{2} \\
& \quad \times \frac{1-2 \cos \left[\left(\varepsilon_{k \mu}-\varepsilon_{e v}\right) t / \hbar\right] e^{-K_{v} t / 2}+e^{-K_{v} t}}{\left(\varepsilon_{k \mu}-\varepsilon_{e v}\right)^{2}+\left(K_{v} \hbar / 2\right)^{2}} \\
& \quad+\operatorname{Re}\left[\sum_{k} \sum_{v \neq v^{\prime}}\langle v \mid \mu\rangle\left\langle\mu \mid v^{\prime}\right\rangle A_{e v}(t=0) A_{e v^{\prime}}(t=0)\right. \\
& \quad \times\left(\frac{1-e^{\left[i\left(\varepsilon_{k \mu}-\varepsilon_{e v^{\prime}}\right)-K_{\nu} \hbar / 2\right] t / \hbar}}{i\left(\varepsilon_{k \mu}-\varepsilon_{e v^{\prime}}\right)-K_{v^{\prime}} \hbar / 2}\right) \\
& \left.\quad \times\left(\frac{1-e^{\left[i\left(\varepsilon_{k \mu}-\varepsilon_{e v}\right)-K_{v} \hbar / 2\right] t / \hbar}}{i\left(\varepsilon_{k \mu}-\varepsilon_{e v}\right)-K_{v} \hbar / 2}\right)\right] .
\end{aligned}
$$

Performing the summation over $k$ states by a continuum integration we obtain the following limit in the absence of a vibrational wavepacket,

$$
\left|A_{\mu}(t)\right|^{2}=\frac{\sum_{\nu}|\langle v \mid \mu\rangle|^{2}\left|A_{e v}(t=0)\right|^{2}\left(1-e^{K_{-\nu} t}\right)}{\sum_{\mu}|\langle v \mid \mu\rangle|^{2}} .
$$

The total rise of probability in the product state is obtained by summing over all final vibrational states $|\mu\rangle$ and the above expression reduces to

$$
\left.\sum_{\mu} A_{\mu}(t)\right|^{2}=\left.\sum_{\nu} A_{e v}(t=0)\right|^{2}\left(1-e^{K_{-\nu} t}\right) .
$$

Thus, the smooth rise in population is not single exponential if the decay proceeds from several initial vibrational states, unless one invokes the wide-band limit, wherein $\sum_{\mu}|\langle\nu \mid \mu\rangle|^{2}=1$ and $K_{\nu} \equiv K$. In the wideband limit, the rate of rise of probability is thus the same for all vibrational levels of the ionized molecule. Several experimental investigations of photoinduced heterogeneous ET utilizing pump-probe methods have measured the total probability of the cationic product state ${ }^{19}$ given by (11), but the vibrationally resolved probability of (9) has not been measured as yet. The population dynamics of individual vibrational levels does not yield new timescale in the wide-band limit. Accordingly, one observes in figure 4 an overall rate for the rise of population among the various vibrational levels that is similar to that of the rise of total population in the cationic product state. By contrast, the rise of population of individual vibrational levels carries not only the imprint of vibrational coherence, which appears as steps at the vibrational frequency, but also the phase relations among the individual vibrational levels. Similar to injection from a single vibrational level into the electronic QC, the excited state vibrational wavepacket transfer of population into specific vibrational levels of the molecular ion depends on its location on the excited state PES. As figure 4 indicates, population injection into the lowest and the sixth vibrational levels, for instance, is roughly $\pi$ out of phase, because during the time when population rises in one state, it remains stationary in the other. As mentioned earlier, this is because the PES crossings between the electronic states in the QC that contribute most to these two final vibrational levels and the excited state, are located close to the opposite ends of the classical turning point of the excited state vibrational wavepacket. On the other

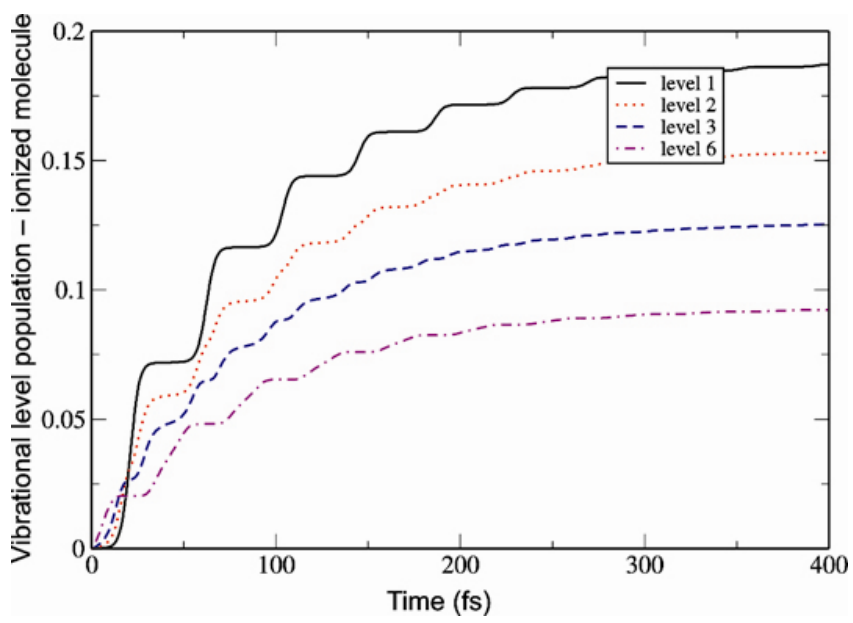

Figure 4. Rise of population of selected vibrational levels of the ionized molecule obtained from (9). All parameters are as in figure 3 . 
hand, as the PES of the QC electronic state that dominates the contribution to the third level crosses the excited state PES close to its mid-point, population is injected into it twice during every period of the excited state vibrational wavepacket.

It is possible to extend (7) and (9) to analytically include: (i) several vibrational modes, similar to what has been done in the expression for the decay, ${ }^{4}$ (ii) vibrational coordinate dependent electronic coupling (which would modify only the Franck-Condon overlap terms in (7)), and (iii) non-uniform density of states. ${ }^{4}$ When a significant part of the injected distribution is located at, and consequently distorted by, the band edge, (4), (7) and (9) fail to describe the dynamics accurately.

\section{Conclusion}

In summary, the essential physics underlying the process of electron injection from a discrete electronic state to a continuum of substrate levels has been discussed within an analytical model. This process is fundamental to several areas of interfacial science and its analysis leads to a simple and yet complete description of the injection process from the very early times to the late times, when the injected distribution reduces to the well-known form described by the Wigner-Weisskopf and FanoAnderson models. Furthermore, the analysis has been extended to include molecular vibrational degrees of freedom that could be either coherently or incoherently excited via suitable laser pulses. Importantly, the analysis brings to fore new physics in the transfer of probability to the various continuum levels, which is manifested as a slower rise or as transient quantum beats within an appropriate energy window. It has been suggested that energy and time-resolved methods such as 2PPE can be utilized $^{18}$ to observe the predictions of the theory presented here.

In the present work, we have focused specifically on the electron injection problem, but the model developed is more general, and could be applied to other problems that involve a discrete state coupled to a quasi-continuum. These include internal conversion and intersystem crossing in gas phase polyatomic excited states, conductance through semiconductor- based molecular junctions, and atomic (or molecular) excitation into a dense manifold of high-lying Rydberg states. It would be interesting for future research to apply the model to these and other problems.

\section{Acknowledgements}

T S is grateful to the US National Science Foundation (Grant No. CHE-0616927 and MRSEC Grant No. DMR-0520513) for support.

\section{References}

1. Miller R D, McLendon G L, Nozik A J, Schmickler W and Willig F 1995 In Surface electron transfer processes (New York: VCH)

2. Guo H, Saalfrank P and Seideman T 1999 Prog. Surf. Sci. 62239

3. Nitzan A and Ratner M A 2003 Science 3001384

4. Ramakrishna S, Willig F and May V $2001 \mathrm{~J}$. Chem. Phys. 1152743

5. Zimmermann C, Willig F, Ramakrishna S, Burfeindt B, Pettinger B, Eichberger R and Storck W $2001 \mathrm{~J}$. Phys. Chem. B105 9245

6. Benko G, Kallioinen J, Korppi-Tommola E I, Yartsev A P and Sundström V $2002 \mathrm{~J}$. Am. Chem. Soc. 124 489

7. Domcke W 1991 Phys. Rep. 20897

8. Kyrölä E and Eberly J H 1985 J. Chem. Phys. 82 1841

9. Sebastian K L and Tachiya M 2006 J. Chem. Phys. 124064713

10. Ramakrishna S, Willig F and May V 2000 Phys. Rev. B62 R16330

11. Mohr J, Schmickler W and Badiali J P 2006 Chem. Phys. 324140

12. Tsivlin D V, Willig F and May V 2008 Phys. Rev. B77 035319

13. Weisskopf V F and Wigner E 1930 Z. Phys. 6354

14. (a) Anderson P W 1961 Phys. Rev. 124 41; (b) Fano U 1961 Phys. Rev. 1241866

15. Haug H and Koch S W 1993 Quantum theory of the optical and electronic properties of semiconductors (Singapore: World Scientific)

16. Maschke K, Thomas P and Göbel E O 1991 Phys. Rev. Lett. 672646

17. Petek H and Ogawa S 1997 Prog. Surf. Sci. 56239

18. Gundlach L, Ernstorfer R and Willig F 2007 Prog. Surf. Sci. 82355

19. Asbury J B, Hao E, Wang Y, Ghosh H H and Lian T 2001 J. Phys. Chem. B105 4545 